\title{
STOCHASTIC ANALYSIS OF BUBBLE AND PARTICLE MOTIONS IN A 2-D THREE-PHASE REACTOR
}

\author{
Ryuji KIKUCHI, Atsushi TSUTSUMI \\ AND Kunio YOSHIDA \\ Department of Chemical System Engineering, The University of \\ Tokyo, Tokyo 113
}

Key Words: Three-Phase Reactor, Bubble Dynamics, Particle Motion, Optical Probe, Power Spectrum

\begin{abstract}
The bubble and particle frequencies in a 2-D gas-liquid-solid three-phase reactor were measured with a novel optical transmittance probe using a narrow laser beam. The signals for the gas and solid phases were extracted separately based on the differences in the shape and voltage level of the signals. The power spectral densities of the extracted time series data for both phases were then calculated. All the power spectra were found to exhibit a continuous spectrum and exponential decay in the high-frequency region. Comparison between the power spectra of both phases indicates that these can be classified into three frequency regions: the low frequency region where most of the peaks were found to be common to both the gas and solid phases and no component intrinsic to the solid phase was found; the frequency region between 10 and $35 \mathrm{~Hz}$ where the components intrinsic to each of the gas and solid phases were found; and the region above $35 \mathrm{~Hz}$ where the components intrinsic to the solid phase were only observed. No significant change of the power spectrum intrinsic to the gas phase was observed with the gas velocity, whereas the component of the power spectrum of the solid phase, especially in the high-frequency region, showed a dependence on the gas velocity. These results indicate that although bubble rising motion affects the dominant frequency of the solid phase, the bubble dynamics were almost independent of the local solid flow structure.
\end{abstract}

\section{Introduction}

Multi-phase systems exhibit very complex hydrodynamic behavior due to the influence of a variety of phenomena such as jetting and bubbling of the fluidizing medium and the motion of fluidized particles. Many attempts to analyze the pressure fluctuation have been made in order to understand the hydrodynamic behavior in gas-liquid, gas-solid and gas-liquid-solid systems. Hydrodynamic behavior has been regarded as stochastic; however, recent results have proved it to be essentially chaotic. Since pressure fluctuations in the systems are considered to be the direct consequences of complicated phenomena inside a reactor, the pressure fluctuation behavior has been studied through statistical methods such as probability density, autocorrelation and power spectral density functions (Lirag and Littman, 1971, Fan et al., 1981, Nishiyama et al., 1987, Yashima et al., 1992). Lirag and Littman (1971) found that in a gassolid fluidized bed the pressure fluctuation was caused by fluctuations of the bed height due to bubbles escaping at the surface of the bed. They also found the presence of a periodic component in the fluctuations by statistical analysis. Fan et al. (1981) investigated the effects of gas velocity, bed height,

* Received on March 7, 1996. Correspondence concerning this article should be addressed to A. Tsutsumi. particle size and distributor design on the major frequency and amplitude of the pressure fluctuations in a gas-solid fluidized bed. They concluded that the pressure fluctuations were caused by the motion and formation of bubbles in the bed. Nishiyama et al. (1987) analyzed pressure fluctuations in a three-phase fluidized bed by a stochastic approach and classified the flow mode prevailing within a three-phase fluidized bed into three regions: three-phase fluidized region, transient region and bubbling flow region. Yashima et al., (1992) investigated pressure fluctuations in a three-phase fluidized bed by power spectral density functions and stated that three successive regions exist in the flow direction of bubbles and the liquid medium: the entrance region near the bottom, followed by the main region, which in turn is followed by the transition region. The spectral density of the entrance region appears to be of Gaussian noise; that of the main region, chaotic; and that of the transition region, stochastic. Based on the fractal concepts, Fan et al., (1990) analyzed pressure fluctuations in a three-phase fluidized bed using Hurst's rescaled range analysis. They investigated the effects of superficial gas velocity, liquid velocity and probe height on the Hurst exponent, $H$, and stated that the fractional Brownian motion model was capable of modeling long-term persistence in the pressure fluctuations. Kwon et al., (1994) measured pressure fluctuations and bubble-chord lengths in 
three-phase fluidized beds. They determined $H$ for a time series data of the pressure fluctuations, and correlated $H$ in terms of the operational variables and the geometrical features of the beds.

Pressure fluctuations are induced by the complex hydrodynamic behavior of a system. Therefore, stochastic analysis of pressure fluctuations is a powerful method in revealing the hydrodynamics of multi-phase flows. However, it seems difficult to determine the governing phenomena responsible for pressure fluctuations because pressure fluctuations are affected in a complex manner by the motion of the fluidizing medium, bubbles and particles. In fact, there is as yet no clear explanation for the physical processes that produce pressure fluctuations. Most pressure fluctuation analysis has been devoted to the classification of flow regimes. In addition, pressure fluctuations, which are induced at different locations, can be transmitted in the bed medium and be superimposed on each other. Thus, it is difficult to extract the local features and behavior of multi-phase systems from the pressure fluctuation signals. Therefore, even though pressure fluctuations can be characterized successfully in a system, the dynamics of the system remains uncertain until the governing phenomena responsible for the pressure fluctuations have been identified.

In this study, a novel optical transmittance probe with a thin laser beam was developed to detect both bubble and particle motions separately in gasliquid-solid systems. A power spectral analysis of the optical signals obtained in the three-phase flow in a 2-D column was performed to characterize the stochastic behavior of both solid and gas phases.

\section{Experimental}

A schematic diagram of the bubble and particle frequency measuring system is shown in Fig. 1. Experiments were conducted in a two-dimensional transparent acrylic resin column of $1.5 \mathrm{~m}$ height, $0.56 \mathrm{~m}$ width and $0.01 \mathrm{~m}$ nominal gap thickness. Gas was fed into the column from a cylinder through 13 nozzles of $4.0 \mathrm{~mm}$ i.d. which were mounted on the bottom at intervals of $30 \mathrm{~mm}$. Tap water and nitrogen were used as the liquid and gas phases, respectively. The solid particle was $780-\mu \mathrm{m}$ Nylon beads with a density of $1150 \mathrm{~kg} / \mathrm{m}^{3}$. The volume fraction of the solids was 0.01 . The superficial gas velocity was varied in the range of $1.2-7.8 \mathrm{~cm} / \mathrm{s}$.

A set of plano-convex lenses was utilized to narrow the initial $1-\mathrm{mm}$ circular $\mathrm{He}-\mathrm{Ne}$ laser emission to $0.1 \mathrm{~mm}$. The narrowed laser beam was projected onto the center of the column wall $30 \mathrm{~cm}$ above the bottom. The intensity of the light passing through the column was measured by a phototransistor located behind the bed opposite to the laser beam and

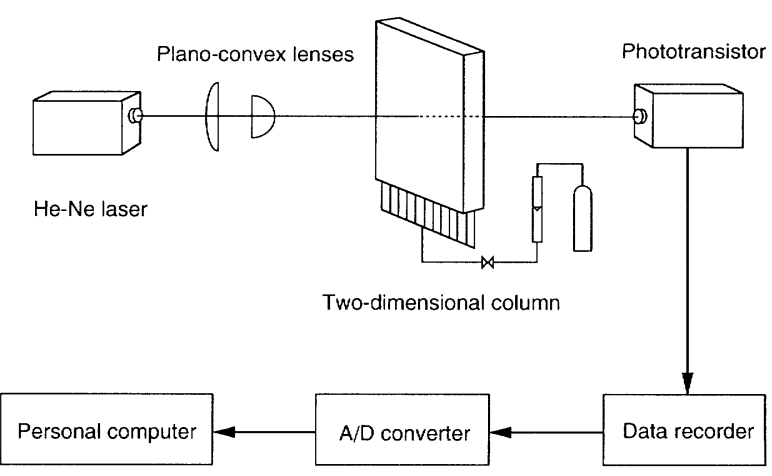

Fig. 1 Schematic diagram of bubble and particle frequency measuring system

converted to a voltage signal. The voltage output was recorded on a data recorder and digitized with an AD converter at 2500 points per second. A typical sample size was 32760 points.

On the basis of the differences in the shape and voltage level, each signal for the gas and solid phases was extracted separately from the time series data obtained under three-phase conditions. Thus, the extracted time series contains the signal for either gas or solid phase. Spectral density functions were calculated for each of the extracted time series data of particle and bubble frequencies.

To confirm the validity of the method of measuring bubble and particle frequencies, bubble passage in a nitrogen-water-Nylon beads system was observed with a 16-mm high-speed movie camera operated at a rate of 1000 frames per second. The change in voltage output from the phototransistor as viewed on an oscilloscope was superimposed on the high-speed cine film.

\section{Results and Discussion}

Typical output signals for solid-liquid and gasliquid systems are given in Figs. 2 and 3, respectively. When either a bubble or a particle intercepts the laser beam, the voltage output decreases. In the solidliquid system, wedge-shaped signals, each of which represents the passage of a solid particle, were registered. The constancy of the slope of each signal is observable. This is ascribable to the rigidity of a solid surface. The minimum voltage of each signal never went below $0.5 \mathrm{~V}$. On the other hand, in the gas-liquid system, most bubbles were detected as broad signals. The minimum voltage of the signal for bubbles was mostly lower than that for particles. A fluctuating structure was developed and several small peaks superimposed on the main peak were observed. This is considered to be due to wobbling of the bubble surface as it rises in the bed. The sequence of five pictures in Fig. 4(a)-(e) shows the 


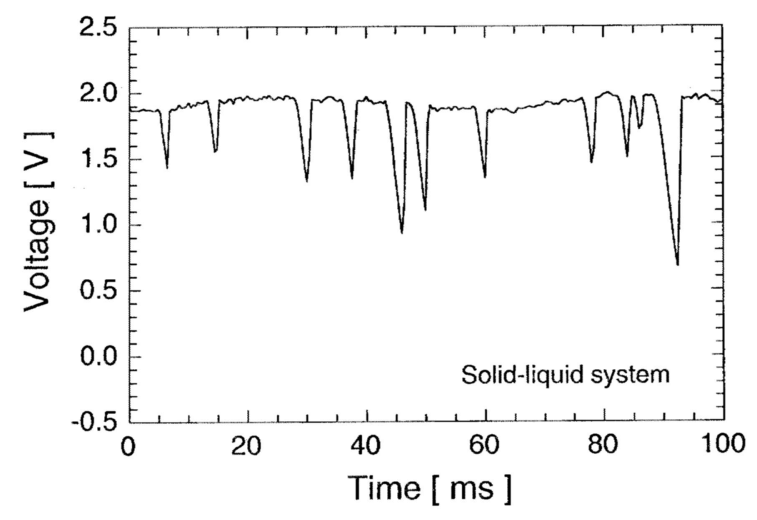

Fig. 2 Typical time series of optical signal in solid-liquid system

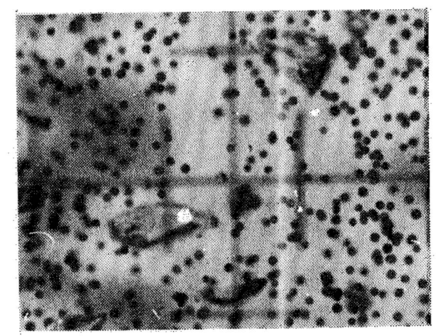

(a) $0 \mathrm{~ms}$

$10 \mathrm{~mm}$

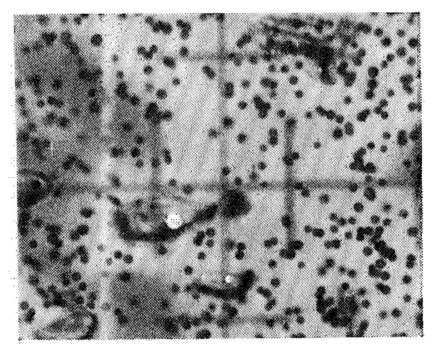

(b) $12 \mathrm{~ms}$

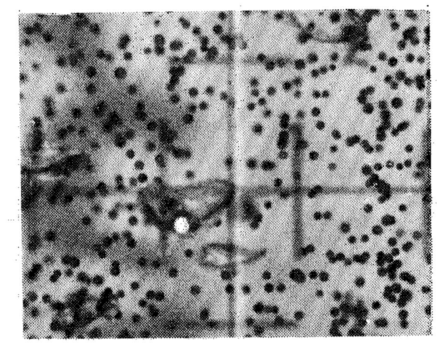

(c) $28 \mathrm{~ms}$

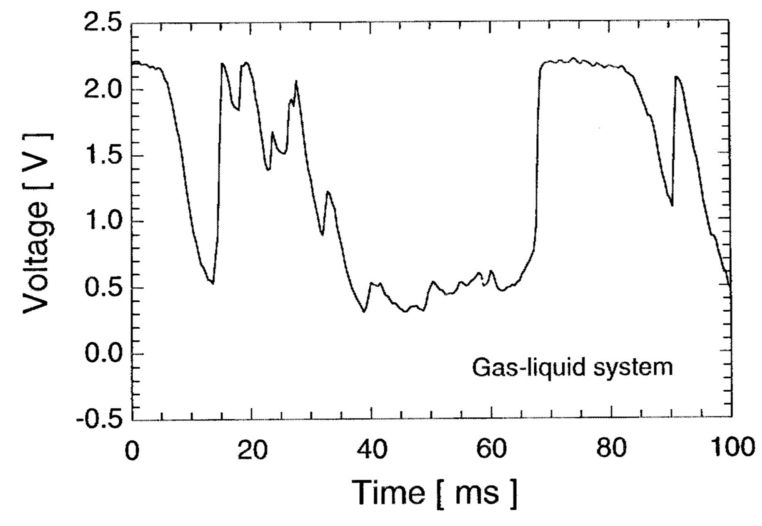

Fig. 3 Typical time series of optical signal in gas-liquid system

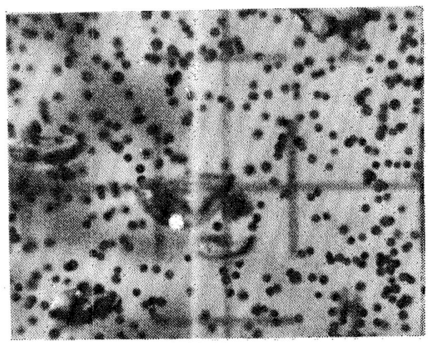

(d) $32 \mathrm{~ms}$

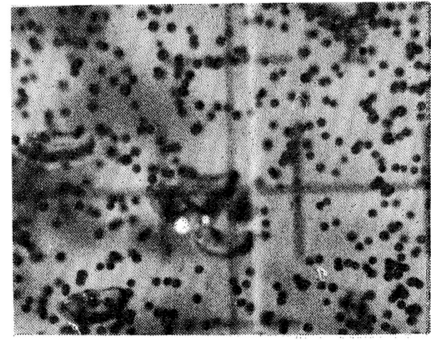

(e) $41 \mathrm{~ms}$

Fig. 4(a)-(e) Photographs showing bubble passage in nitrogen-water-Nylon beads system: White vertical line represents superimposed voltage output

passage of bubbles observed with the high-speed movie camera in the nitrogen-water-Nylon beads system together with the superimposed signal of the optical output from the phototransistor. These photographs were taken in the same direction as the laser beam was projected. The vertical white line represents the superimposed voltage output. The vertical white line shifts to the left in the photographs when the voltage output drops. Figures 4(a) and (e) show the moment when the bubble is just about to come into and go out of contact with the laser beam, respectively. Figures $4(\mathrm{~b})-(\mathrm{d})$ show the change in the voltage output during the bubble passage; the voltage output first drops (Fig. 4(b)), then rises (Fig. 4(c)), and drops again (Fig. 4(d)). It is clear that voltage output drops as soon as a bubble comes into contact with the 

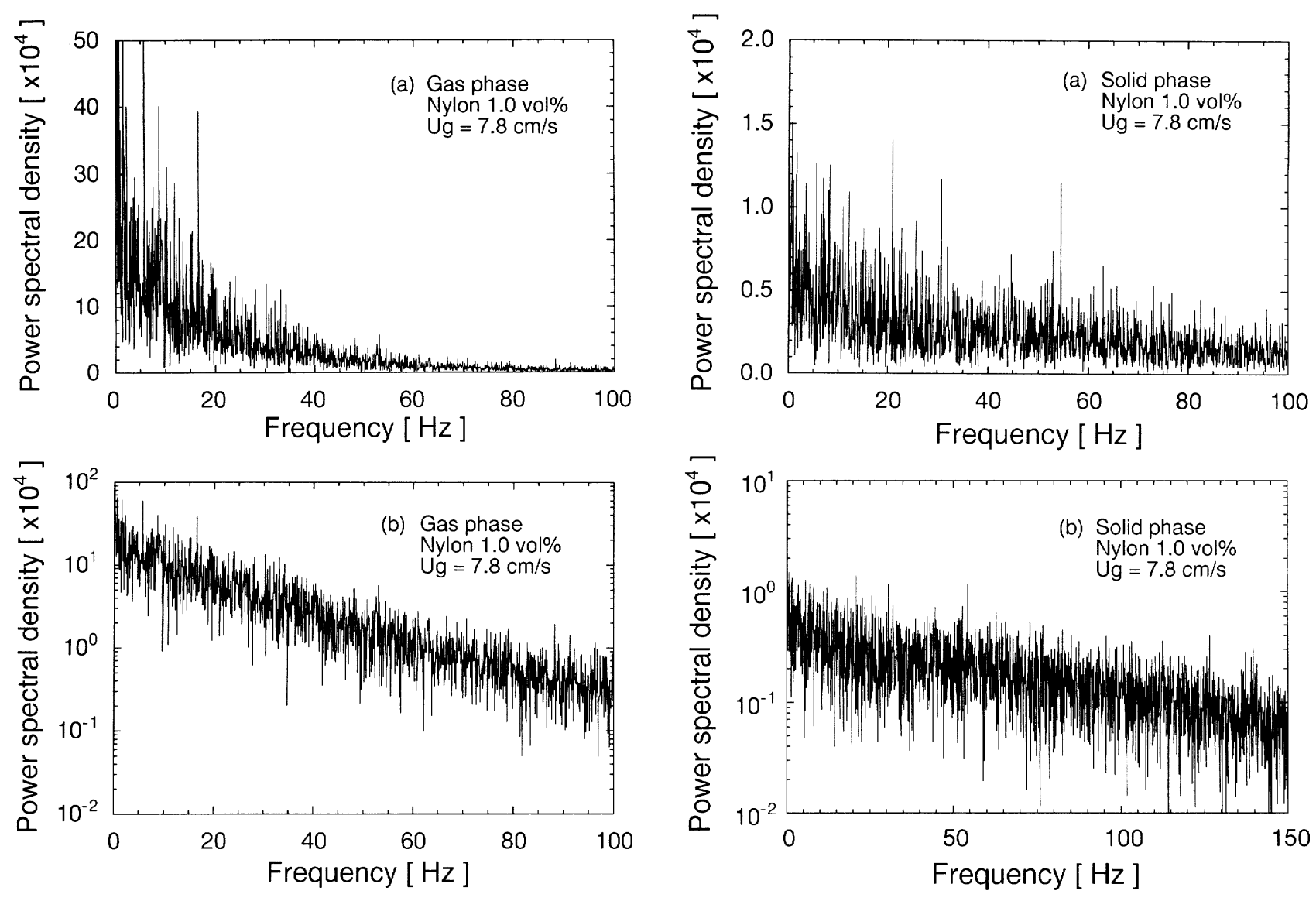

Fig. 5 Typical plots on (a) linear and (b) semilogarithmic scales of a power spectrum of extracted time series data for gas phase

laser beam, then returns to its baseline right after the bubble has passed through the beam. In addition, it is obvious that the voltage output oscillates during the bubble passage and that it does not return to the baseline during the passage. Thus, it is confirmed that each oscillating broad signal corresponds to the passage of a bubble.

Figures 5 and 6 show typical power spectral density functions of the extracted time series data plotted on (a) linear and (b) semilogarithmic scales for the gas and solid phases, respectively, of the nitrogenwater-Nylon beads system. It can be seen that the power spectral density functions for both the phases exhibit a continuous power spectrum with many spikes. All the power spectra of the gas and solid phases were found to show exponential decay up to 80 and $150 \mathrm{~Hz}$, respectively. In general, systems which can be described by deterministic equations with a few variables have power spectra with exponential decay, while stochastic systems exhibit power-law decay (Greenside et al., 1982, Ruelle, 1986, Sigeti and Horsthemke, 1987). Therefore, the appearance of the continuous spectrum and the exponential decay of power spectral density functions in the present study

Fig. 6 Typical plots on (a) linear and (b) semilogarithmic scales of a power spectrum of extracted time series data for solid phase

implies that the hydrodynamic behavior of threephase systems is chaotic.

The power spectral density of the gas phase falls off and drops to a negligible level at above $80 \mathrm{~Hz}$ (Fig. 5a). On the other hand, the peaks in the spectrum of the solid phase are distributed over the high frequency region (Fig. 6a). By comparing the spectra of the gas and solid phases, distinct peaks were assigned to common as well as intrinsic components. In order to identify the distinct peaks, the components whose spectral densities were within $5 \%$ in descending order from the maximum spectral density were extracted for each of the gas and solid phases and the square root of their product at the same frequency was calculated. Figures 7(a)-(c) show the extracted spectra for both the gas and solid phases of the nitrogen-water-Nylon beads system (Figs. 7(a) and (b)) and the square root of the product as a function of frequency (Fig. 7(c)). The distinct peaks were assigned, in reference to the square root of the product, to intrinsic or common components to each phase. The assignments are indicated in Figs. 7(a) and (b). As can be seen, the power spectra can be classified into three frequency regions. At low frequency, most peaks are found to 

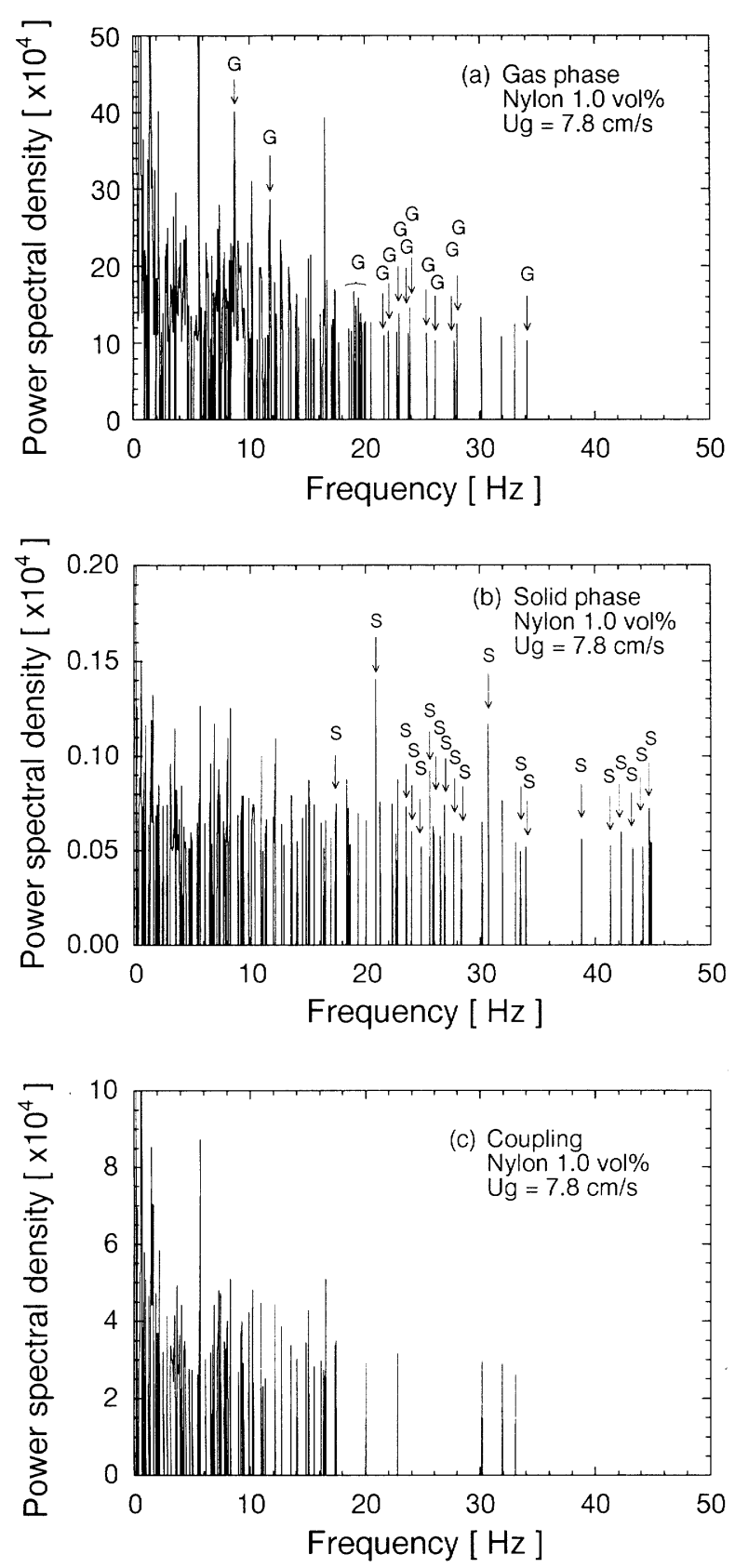

Fig. 7 Assignment of intrinsic and common components to (a) gas and (b) solid phases, (c) square root of the product of the extracted distinct peaks of both phases: in each spectrum, intrinsic components are marked as "G" or " $\mathrm{S}$ " for gas and solid phases, respectively.

be common components to both gas and solid phases. There are a few intrinsic components to the gas phase but none to the solid phase. On the other hand, above $35 \mathrm{~Hz}$ the spectrum of the gas phase has no distinct peak; only the intrinsic components of the solid phase are observed. In the frequency region between 10 and $35 \mathrm{~Hz}$, intrinsic components were found in the spectra for both phases. These characteristics of the power spectral density functions were observed for all systems in the present experiments.

The turbulent fluctuation in a three-phase system is caused primarily by bubble rising motion. Rising bubbles induce vortical and chaotic motion in the liquid-solid medium, leading to the prevailing macroscopic flow structure of the system. In addition, solid particles are entrapped in the bubble wakes and carried. It can be considered, therefore, that the primary frequency induced by the bubble rising motion is common to both gas and solid phases and the same as a bubble passing frequency. At a gas velocity of $7.8 \mathrm{~cm} / \mathrm{s}$, the average frequency of bubble passing across the laser beam was $16.8 \mathrm{~Hz}$ for the nitrogen-water-Nylon beads system. Hence, the distinct peaks common to both phases in the lowfrequency region between 0 and $16.8 \mathrm{~Hz}$ in Figs. 7(a) and (b) are attributable to the bubble rising motion. In general, bubble size distributions follow a logarithmic normal distribution (Akita and Yoshida, 1974). Thus, large bubbles have lower frequencies than small bubbles because of the small population. It is highly probable that the lower-frequency components stem from the rising motion of large bubbles.

Bubble and solid motions relating to the wake shedding phenomena give rise to one of the basic frequencies of the gas and solid phases. The wake shedding frequency can be estimated to be between 16 and $19 \mathrm{~Hz}$ through the following correlation (Fan and Tsuchiya, 1990) for the Strouhal number $\operatorname{Sr}\left(=f_{b} d_{b} /\right.$ $\left.U_{b}\right)$ with the Reynolds number $\operatorname{Re}_{b}\left(=\rho U_{b} d_{b} / \mu\right)$ :

$$
\frac{1}{S r}=4840 \operatorname{Re}_{b}{ }^{-1.02}+1.55
$$

where the bubble diameter was calculated using the following empirical correlation (Fukuma et al., 1987):

$$
d_{b}=0.59\left[U_{g}\left(1-\varepsilon_{g}\right) / \varepsilon_{g}\right]^{2} g^{-1}
$$

Hence, the dominant peaks in the power spectra of both gas and solid phases at around $17 \mathrm{~Hz}$ can be assigned to the fluctuations induced by wake shedding.

The frequency of bubble or particle passing through the laser beam is one of the fundamental frequencies of the gas or solid phase, respectively. One can estimate the frequencies with $U_{b} / d_{b}$ and $v / d_{p}$, where $d_{b}$ and $d_{p}$ are mean bubble and particle diameters, and $U_{b}$ and $v$ represent bubble rising velocity and particle settling velocity, respectively. For Nylon beads with a diameter of $780 \mu \mathrm{m}$, the value of $v / d_{p}$ was estimated to be around $33 \mathrm{~Hz}$. The average particle passing frequency obtained from the optical signal was $19.5 \mathrm{~Hz}$ at a gas velocity of $7.8 \mathrm{~cm} /$ s. Hence, in the power spectrum of the solid phase 
the contribution of frequencies between 19.5 and $40 \mathrm{~Hz}$ is attributable to the particle passing phenomenon. For the gas phase, the estimated value of $U_{p} /$ $d_{b}$ was about $38 \mathrm{~Hz}$ and the average bubble passing frequency was $16.8 \mathrm{~Hz}$ at a gas velocity of $7.8 \mathrm{~cm} / \mathrm{s}$. Thus, in the spectrum of the gas phase, the peaks in the frequency region between 16.8 and $38 \mathrm{~Hz}$ are assigned to the bubble passing phenomena through the laser beam. There are a few common peaks in the power spectrum of the gas phase in this region. This indicates that despite the bubble rising motion affecting the dominant frequency of the solid phase, the bubble dynamics are almost independent of the local solid flow structure.

Figure 8 shows the effect of superficial gas velocity on the intrinsic and common components of the power spectra for the solid phase: the common and intrinsic components are colored black and red, respectively. It is seen that the number of the common components below $10 \mathrm{~Hz}$ and at around $17 \mathrm{~Hz}$ increases with the gas velocity. At a gas velocity of $2.3 \mathrm{~cm} / \mathrm{s}$, fewer common components in the low frequency region are found than at other gas velocities. In addition, at around $17 \mathrm{~Hz}$, that is, the wake shedding frequency, there are a few common and intrinsic components. At this gas velocity the flow field in the bed is the homogeneous bubbly flow where small bubbles are dispersed and the bubble population is small. Hence, since the solid particles entrapped in the bubble wakes are small, both the spectral density and the number of the components at around $17 \mathrm{~Hz}$ and below $10 \mathrm{~Hz}$ are small. On the other hand, as the gas velocity increases, the bubble size and population increase and the interaction between bubbles becomes significant, leading to the coalescence and breakup of bubbles. Further increase in the gas velocity causes transition from the homogeneous bubbly flow to the churn-turbulent flow. Thus, as the gas velocity increases, entrapment and shedding of solid particles in the bubble wakes becomes frequent, resulting in the increase in the number of components at around $17 \mathrm{~Hz}$.

In the frequency region between 25 and $100 \mathrm{~Hz}$, many intrinsic components of the solid phase are observed for all the gas velocities. No intrinsic component is observable above $100 \mathrm{~Hz}$. As mentioned above, the components intrinsic to the solid phase in this region are ascribed to the particle passing phenomenon. The frequency of 25 to $100 \mathrm{~Hz}$ corresponds to a particle velocity of 2 to $8 \mathrm{~cm} / \mathrm{s}$. It is considered, therefore, that the intrinsic components in this frequency region represent the frequency of the solid particles suspended in the liquid medium and following the liquid flow induced by the motion of rising bubbles. At a gas velocity of $2.3 \mathrm{~cm} / \mathrm{s}$ more than half of the intrinsic components exist between 25 and $45 \mathrm{~Hz}$. This indicates that in the homogeneous
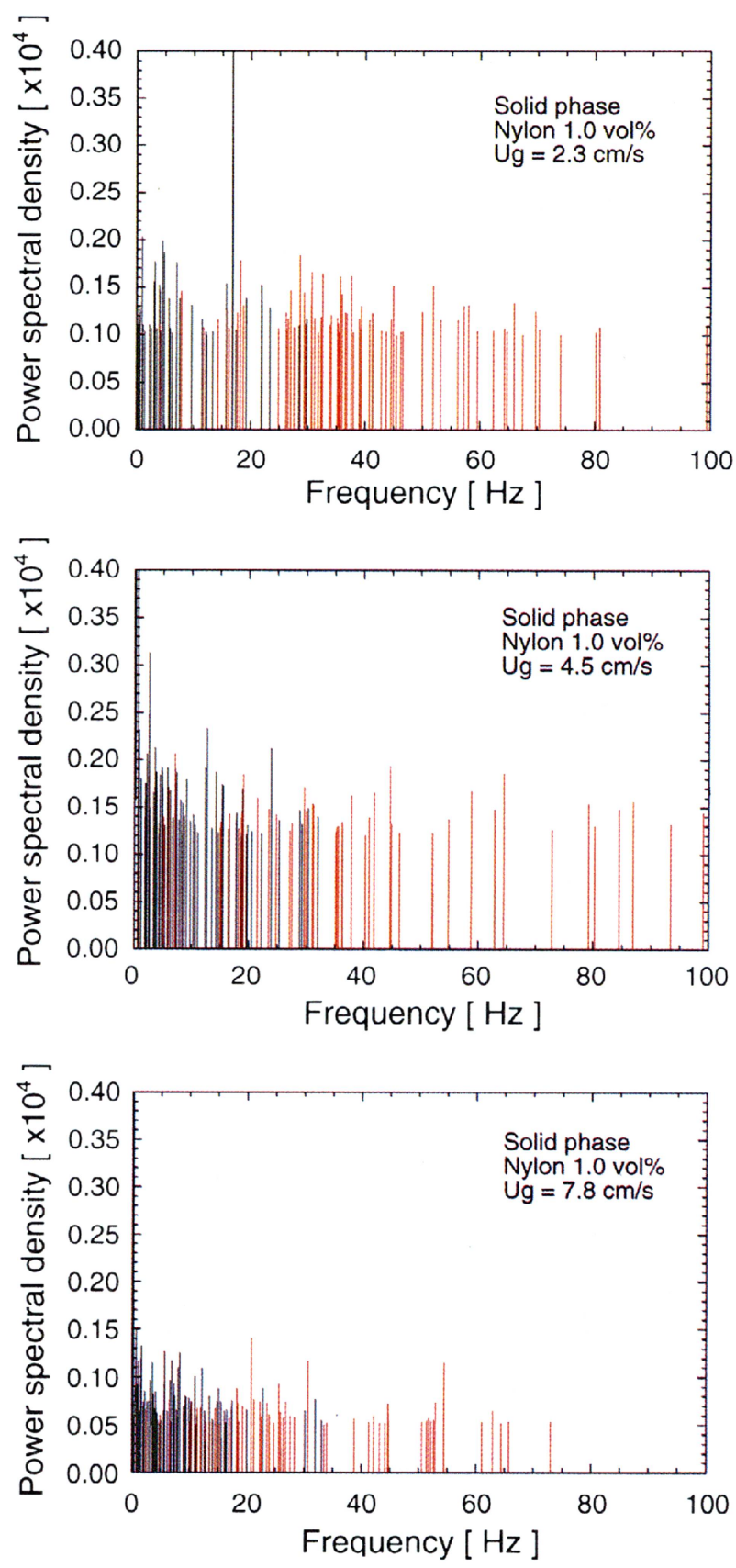

Fig. 8 Effect of superficial gas velocity on intrinsic and common components of the power spectra for solid phase: common and intrinsic components to solid phase are represented by black and red spikes, respectively

bubbly flow regime the velocities of the liquid flow induced by the motion of small bubbles are small and most solid particles are suspended in the liquid flow. At a gas velocity of $4.5 \mathrm{~cm} / \mathrm{s}$, the intrinsic components are distributed over a wide range of frequencies from 25 to $100 \mathrm{~Hz}$. At this gas velocity, the flow pattern changes from the homogeneous bubbling to the churn-turbulent regime. Since bubble coalescence and breakup occur frequently during this transition, 

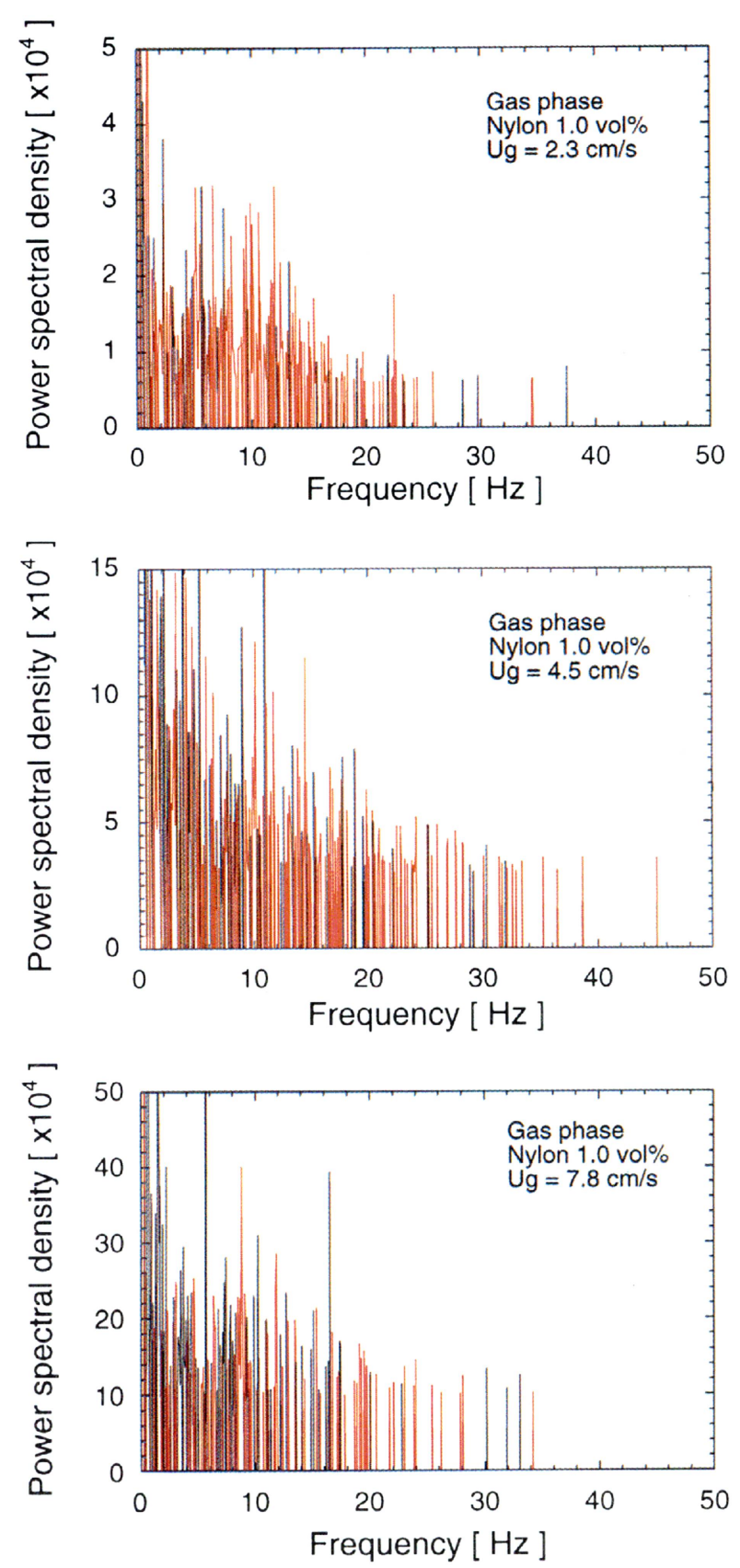

Fig. 9 Effect of superficial gas velocity on intrinsiç and common components of the power spectra for gas phase: common and intrinsic components to gas phase are represented by black and red spikes, respectively

the liquid flow in the bed becomes complex, thereby leading to a wide distribution of the intrinsic components of the solid phase. At a gas velocity of $7.8 \mathrm{~cm} / \mathrm{s}$, the intrinsic components of the solid phase are discretely distributed at around 43,52 and $64 \mathrm{~Hz}$. In the churn-turbulent flow regime, bubble coalescence takes place to form large bubbles with high rising velocities. Because of these high velocities, large bubbles tend to move upward more linearly than
Table 1 Assignment of power spectra

\begin{tabular}{lc}
\hline \multicolumn{1}{c}{ Phenomena } & Frequency $[\mathrm{Hz}]$ \\
\hline $\begin{array}{l}\text { Bubble rising motion } \\
\text { Large bubble }\end{array}$ & $\begin{array}{r}0-20 \\
<10\end{array}$ \\
\hline $\begin{array}{l}\text { Bubble wobbling } \\
\text { Wake shedding }\end{array}$ & $16-25$ \\
\hline Particle settling & $>25$ \\
\hline
\end{tabular}

small bubbles. Hence, solid particles can be considered to follow the coherent flow structures in the liquid phase organized by the upward motion of bubbles, thus generating the discrete distribution of the intrinsic components of the solid phase.

Figure 9 shows the effect of superficial gas velocity on the intrinsic and common components of the power spectra for the gas phase: the common and intrinsic components are colored black and red, respectively. Neither an intrinsic nor a common component is seen above $50 \mathrm{~Hz}$. As is stated in the discussion with the solid phase spectra, a few common components at around $17 \mathrm{~Hz}$ are observed at a gas velocity of $2.3 \mathrm{~cm} / \mathrm{s}$, and the number of the common components increases with the gas velocity. The components in the low-frequency region, which are assigned to the motion of large bubbles, increase with the gas velocity. This tendency agrees well with the observation that bubble coalescence occurs to form large bubbles as the gas velocity increases.

As the gas velocity increases, the intensity of spectral densities of the intrinsic and common components for the gas phase increase. However, no significant change appears in the distribution of the intrinsic and common components for the gas phase. This indicates that the distribution of the time width and interval of bubble signals, which manifests the characteristics of bubble dynamics during the period to be analyzed, does not change significantly with gas velocity. It can be therefore considered that the dynamic characteristics of bubble motion during the period to be analyzed are not affected by the local solid and liquid flows, which are primary influenced by bubble rising motion.

The assignment of the power spectra discussed above is summarized in Table 1.

\section{Conclusion}

A novel optical transmittance probe was developed to discriminate between the gas and solid phases in three-phase systems. In accordance with the differences in the shape and voltage level, signals for the gas and solid phases were distinguished and extracted separately from the time series data obtained under three-phase conditions. 
In the stochastic analysis of the signals, power spectral density functions for both the gas and solid phases were observed to exhibit continuous spectrum and exponential decay up to 80 and $150 \mathrm{~Hz}$, respectively. In addition, it was found that the power spectra of the gas and solid phases can be classified into three frequency regions. In the low-frequency region, most of the distinct peaks were found to be the components common to both gas and solid phases, which were assigned to bubble passing phenomena. No component intrinsic to the solid phase was detected. In the frequency region between 10 and $35 \mathrm{~Hz}$, the components intrinsic to each of the gas and solid phases exist. The distinct peaks at around $17 \mathrm{~Hz}$ were assigned to the fluctuations of bubbles induced by wake shedding phenomena. Above $35 \mathrm{~Hz}$ the components intrinsic to the solid phase were observed, which were assigned to particle passing in the liquid flow.

As the superficial gas velocity increases, a distribution of the components intrinsic to the solid phase changes significantly, especially in the highfrequency region. On the other hand, a distribution of the components intrinsic to the gas phase changes slightly. These results indicate that the timeaveraged characteristics of bubble dynamics are independent of the local solid and liquid flows.

$\begin{array}{llr}\text { Nomenclature } & \\ d_{b} & =\text { mean bubble diameter } & {[\mathrm{m}]} \\ d_{p} & =\text { mean particle diameter } & {[\mathrm{m}]} \\ f_{b} & =\text { wake shedding frequency } & {[\mathrm{Hz}]} \\ \operatorname{Re}_{b} & =\text { bubble Reynolds number }\left(=\rho U_{b} d_{b} / \mu\right) & {[-]} \\ S r & =\text { Strouhal number }\left(=f_{b} d_{b} / U_{b}\right) & {[-]} \\ U_{b} & =\text { bubble rising velocity }\left(=U_{g} / \varepsilon_{g}\right) & {[\mathrm{m} / \mathrm{s}]} \\ U_{g} & =\text { superficial gas velocity } & {[\mathrm{m} / \mathrm{s}]}\end{array}$

$v \quad=$ particle settling velocity $\quad[\mathrm{m} / \mathrm{s}]$

$\varepsilon_{g} \quad=$ gas holdup $\quad[-]$

$\mu \quad=$ viscosity of liquid $\quad[\mathrm{Pa} \cdot \mathrm{s}]$

$\rho \quad=$ density of liquid $\quad\left[\mathrm{kg} / \mathrm{m}^{3}\right]$

Literature Cited

Akita, K. and F. Yoshida; "Bubble Size, Interfacial Area, and Liquid-Phase Mass Transfer Coefficient in Bubble Columns," Ind. Eng. Chem. Process Des. Dev., 13, 84-91 (1974)

Fan, L.-S. and K. Tsuchiya; Bubble Wake Dynamics in Liquids and Liquid-Solid Suspensions, p. 132, Butterworth-Heinemann, Stoneham, MA, U. S. A. (1990)

Fan, L.T., T.C. Ho, S. Hiraoka and W.P. Walawender; "Pressure Fluctuations in a Fluidized Bed," AIChE J., 27, 388-396 (1981)

Fan, L.T., D. Neogi, M. Yashima and R. Nassar; "Stochastic Analysis of a Three-Phase Fluidized Bed: Fractal Approach," AIChE J., 36, 1529-1535 (1990)

Fukuma, M., K. Muroyama and A. Yasunishi; "Properties of Bubble Swarm in a Slurry Bubble Column," J. Chem. Eng. Japan, 20, 28-33 (1987)

Greenside, H.S., G. Ahlers, P.C. Hohenberg and R.W. Walden; "A Simple Stochastic Model for the Onset of Turbulence in Rayleigh-Benard Convection," Physica, 5D, 322-334 (1982)

Kwon, H.W., Y. Kang, S.D. Kim, M. Yashima and L.T. Fan; "Bubble-Chord Length and Pressure Fluctuations in ThreePhase Fluidized Beds," Ind. Eng. Chem. Res., 33, 1852-1857 (1994)

Lirag, R.C. and H. Littman; "Statistical Study of the Pressure Fluctuations in a Fluidized Bed," AIChE Symp. Ser., 67, 11-22 (1971)

Nishiyama, M., T. Kawano, N. Ototake and N. Yutani; "Static Pressure Fluctuations and Flow Mode in a Three-Phase Fluidized Bed," Kagaku Kogaku Ronbunsyu, 13, 348-354 (1987)

Ruelle, D.; "Resonances of Chaotic Dynamical Systems," Phys. Rev. Lett., 56, 405-407 (1986)

Sigeti, D. and W. Horsthemke; "High-Frequency Power Spectra for Systems Subject to Noise," Phys. Rev. A, 35, 2276-2282 (1987)

Yashima, M., R. Nassar, L.T. Fan and Y. Kang; "Stochastic Modeling of Pressure Fluctuations in a Three-Phase Fluidized Bed," AIChE J., 38, 629-634 (1992) 